\title{
Molecular motors and stochastic models
}

\author{
Reinhard Lipowsky \\ MPI für Kolloid- and Grenzflächenforschung \\ 14424 Potsdam, Germany *
}

\section{Source:}

in Stochastic Processes in Physics, Chemistry, and Biology; ed. by J.A. Freund and T. Pöschel; Lecture Notes in Physics, Vol. 557, p. 21-31 (Springer, Berlin 2000)

\begin{abstract}
The behavior of single molecular motors such as kinesin or myosin $\mathrm{V}$, which move on linear filaments, involves a nontrivial coupling between the biochemical motor cycle and the stochastic movement. This coupling can be studied in the framework of nonuniform ratchet models which are characterized by spatially localized transition rates between the different internal states of the motor. These models can be classified according to their functional relationships between the motor velocity and the concentration of the fuel molecules. The simplest such relationship applies to two subclasses of models for dimeric kinesin and agrees with experimental observations on this molecular motor.
\end{abstract}

\section{Introduction and overview}

Molecular motors are ubiquitous in living cells. Indeed, it has been recently realized that all transport processes or movements which occur within the cell in a coherent fashion are governed by such motors. Examples are provided by transmembrane transport of ions and macromolecules, regulated adhesion and fusion of membranes, intracellular vesicle transport, cell division, and cell locomotion. $[1]$

In general, one may distinguish several types of motors: (i) pumps which are membrane proteins used to transport ions and small molecules across the membrane $^{\dagger}$; (ii) rotary motors such as the bacterial flagellar motor and $F_{1}-$ ATPase which are again membrane-bound structures; and (iii) linear motors which move along filaments.

In the following, I will focus on linear motors which move on filaments. and which are processive in the sense that they make many steps before they detach from the filament. Such motors are responsible for vesicle transport and for

\footnotetext{
*Email: lipowsky@mpikg-golm.mpg.de

†It is amusing to note that these pumps act as Maxwell's demons [2] who (or which!) are able to sort two types of molecules into two different compartments.
} 
the (re)organization of the cytoskeleton. It has been estimated that a typical eucaryotic cell might contain between fifty and a hundred different types of linear motors [3]. Several superfamilies of molecular motors have been identified. One of these families consists of kinesin and kinesin-related molecules which move along microtubules [4].

During the last couple of years, new experimental techniques have been used in order to measure the performance of these motors on the mesoscopic scale. The most important property which quantifies this performance is the motor velocity. This latter quantity has been studied for three different classes of motor molecules: (i) Dimeric kinesin on microtubules [5, 6, 7, 8, 9]; (ii) Monomeric kinesin on microtubules [10]; and (iii) Myosin V on actin filaments [11].

The functioning of molecular motors has also been studied from a theoretical point of view using different types of motor models $[12,13,14,15,16,17,18$, $19,20,21]$. In fact, the variety of models which can be found in the literature is somewhat confusing. In this short review, I will discuss the present status of the relation between theory and experiment and some recent attempts to clarify this relation.

The paper is organized as follows. First, Section 2 contains a short summary of the experimental information on dimeric kinesin and on the different theoretical models which have been used to describe its motor properties. The nontrivial coupling between the biochemical cycle and the mechanical movement can be studied in nonuniform ratchet models with spatially localized transition rates as defined in Section 3. These models lead to simple relationships between the motor velocity and the fuel concentration, see Section 4, which can be used to classify these models.

\section{Experiment versus theory}

So far, only a few experiments have been reported for monomeric kinesin and for myosin V. Dimeric kinesin, on the other hand, has been experimentally studied in considerable detail. This experimental work has provided several clues to the latter motor, both with respect to its biochemical and geometric features on the molecular scale and with respect to its motor properties which characterize its performance on supramolecular scales.

\subsection{Experiments on dimeric kinesin}

Dimeric kinesin moves on microtubules as illustrated in Fig. 1. The microtubule is a linear filament consisting of 13 protofilaments of tubulin molecules which form a hollow cylinder. Each protofilament represents a 1-dimensional lattice with a lattice constant of $8 \mathrm{~nm}$. The motor consists of two identical amino-acid chains which form two heads. Each kinesin head can act as an ATPase which adsorbs and hydrolyses ATP. In addition, each head can bind to and unbind 
from the microtubule. Thus, each head has an ATP-adsorption domain and a microtubule-binding domain.

All experimental studies are consistent with the view that dimeric kinesin moves in a 'head-over-head' (or 'hand-over-hand') fashion, i.e., by alternating steps in which one head moves forward while the other one remains bound to the tubule. If the motor does indeed advance by this type of stepping motion, the unbound head and the center-of-mass of the motor would move by $16 \mathrm{~nm}$ and by $8 \mathrm{~nm}$, respectively, during each step.

The relative displacement of the kinesin motor against the filament was determined by optical trap experiments. The most direct evidence comes from experiments in which the filament is firmly attached to a solid substrate and the motor molecule is anchored to a bead. This bead is grapped by the optical tweezers and then brought into contact with the filament. In these experiments, one can directly measure the time evolution of the displacement of a single motor molecule (plus the attached bead). From a large number of such displacement-versus-time curves, one obtains average motor properties such as the motor velocity which characterize the motor performance on length scales large compared to the step size.

For dimeric kinesin, the motor velocity has been measured as a function of two control parameters. The first such parameter is provided by the ATP concentration $\Gamma$, i.e., by the concentration of the fuel molecules. The second control parameter is given by the external force $F$ usually applied by the optical trap.

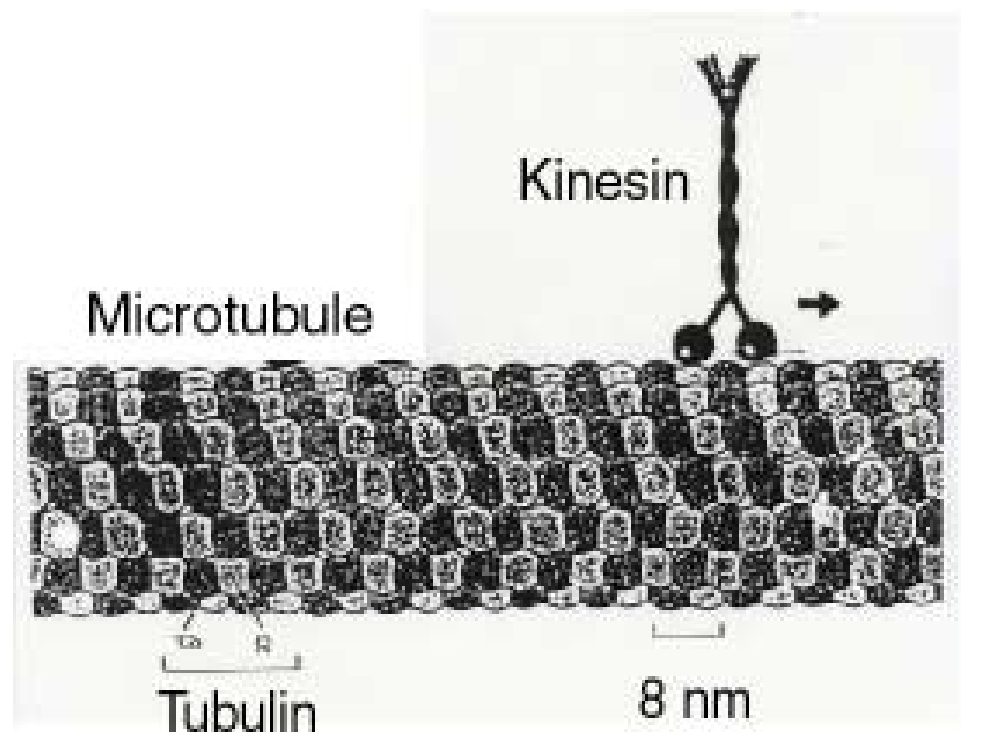

FIG. 1: Bound state of dimeric kinesin on a microtubule - The two heads of the kinesin molecule are bound to two adjacent tubulin segments which are $8 \mathrm{~nm}$ apart. In this cartoon, the stalk of the kinesin, which is about $50 \mathrm{~nm}$, has been reduced compared to the diameter of the microtubule, which is about $25 \mathrm{~nm}$. 
Several experiments have shown that the motor velocity $v$ increases monotonically with the ATP concentration $\Gamma$ and exhibits a saturation behavior. In fact, it has been found for dimeric kinesin that the data for $v=v(\Gamma)$ can be fitted by the functional form $[5,7,8]$

$$
v(\Gamma) \simeq v_{\max } \Gamma /\left(\Gamma_{*}+\Gamma\right)
$$

for vanishing force $F$ (or for small values of $F$ ). Furthermore, if one uses $v_{\max }$ and $\Gamma_{*}$ as $F$-dependent fit parameters, this functional form seems to apply over the whole range of accessible load forces as given by $0 \leq|F| \leq 5.6$. [9]

In the presence of an external load force, the motor velocity is observed to decrease monotonically with increasing load. The precise functional dependence of velocity versus force has been a matter of some controversy. In fact, if the load force is applied to the motor via an attached bead, one generates two force components, one which is tangential to and one which is normal to the filament [7] Since the tether between the bead and the kinesin molecule is not expected to behave as a linear spring, the force applied by the optical trap may not be simply proportional to the tangential force acting on the motor molecule. In view of these uncertainties, I will focus here on the concentration dependence of the motor properties.

\subsection{Different types of theoretical models}

Any molecular motor acts as a processive enzyme, the activity of which requires a certain conformational state of the motor. Since the movement of the motor is directly related to its conformation, one has a nontrivial coupling between this movement and the enzymatic activity of the motor. This coupling represents a nontrivial constraint on the modelling of molecular motors. Several types of motor models have been studied which differ in the basic assumptions about this coupling. One can distinguish three different cases:

(i) Tight-coupling models - In these models, one assumes that the biochemical cycle is independent of the mechanical movement and that the latter movement simply follows this cycle which consists of several conformational or internal states $[12,5,13,7,16,20]$.

(ii) Uniform ratchets - Here, the degrees of freedom which are related to the mechanical movement are taken into account explicitly. The motor can attain several internal states and its position is described by a spatial coordinate, say $x$. However, the enzymatic activity, which leads to transitions between these internal states, is again taken to be independent of the conformation and, thus, of the position $x$ of the motor. Examples for these types of models are ratchets with flashing potentials [14] and with several internal motor states but spatially uniform transition rates $[15,17,19]$.

(iii) Nonuniform ratchets or diffusion-reaction models - As in the uniform case, the motor can attain several internal states and its position is described explicitly but, in addition, one now incorporates the crucial feature that 
the enzymatic activity depends on the spatial position. Thus, these models are characterized by transition rates between the internal states which depend on the spatial coordinate $x$. $[15,19,21]$

Models which are intermediate between case (ii) and (iii) correspond to ratchets with flashing potentials where single potential segments can be switched independently $[18]$.

As far as the concentration dependence of the motor velocity is concerned, these different types of models lead to the following relationships:

(i) Tight-coupling models - It is assumed that the enzymatic activity is governed by Michaelis-Menten kinetics. [5, 7] This implies that the functional dependence of the ATP-hydrolysis rate $\omega$ on the ATP concentration $\Gamma$ is given by $\omega_{\text {hyd }}(\Gamma)=\omega_{\text {sat }} \Gamma /\left(\Gamma_{*}+\Gamma\right)$. It then follows from the tight-coupling assumption and the size $\Delta x$ for a single motor step that the motor velocity $v$ is simply given by $v(\Gamma)=\omega_{\text {hyd }}(\Gamma) \Delta x$. This agrees with the experimentally observed dependence as in (1). It turns out, however, that this functional dependence also holds for a large class of nonuniform ratchets, see below, and, thus, cannot be regarded as strong evidence for tight coupling.

(ii) Uniform ratchets - These models lead to a functional form of the motor velocity $v$ which exhibits a maximum as a function of the transition rates. The same behavior is found for flashing potentials with independent switching of single potential segments. Since the ATP concentration $\Gamma$ enters via these rates, these models also predict that the motor velocity $v=v(\Gamma)$ decreases for large $\Gamma$, in disagreement with the experimentally observed dependence (1).

(iii) Nonuniform ratchets - These ratchets can lead to a motor velocity which increases monotonically with the ATP concentration $\Gamma$. This was first found for ratchets with two internal states and with localized transitions at two spatial positions. [15, 19] We have recently generalized this theoretical framework and introduced nonuniform ratchet models which are characterized by $M$ internal states and by transitions at $K$ spatial locations within one potential period [21]. These models are described in the following Section 3. It is possible to determine and classify the functional dependence $v=v(\Gamma)$ for arbritrary $M$ and $K$, see Section 4. In particular, several classes of $(M, K)$-models can be identified which lead precisely to the hyperbolic form as given by (1).

\section{Nonuniform $(M, K)$ ratchets}

The theoretical framework used here is based on the time evolution of the probability densities $P_{m}(x, t)$ to find the motor particle at center-of-mass coordinate $x$ and in internal state (or level) $m$ which can attain $M$ values $m=1, \ldots, M$. For a given position $x$, each probability density $P_{m}$ may change (i) because of lateral diffusion in state $m$ which leads to lateral currents $J_{m}$ or (ii) becauce of transi- 
tions between the different internal states. $\ddagger$ Therefore, the probability densities $P_{m}$ satisfy the continuity equations $\partial P_{m}(x, t) / \partial t+\partial J_{m}(x, t) / \partial x=I_{m}(x, t)$ with the transition current densities $I_{m}$.

The lateral currents $J_{m}$ depend on the molecular interaction potentials $U_{m}(x)$ and on the external force $F$ which define the effective force potentials

$$
V_{m}(x) \equiv\left(U_{m}(x)-F x\right) / T
$$

where $T$ is the temperature in energy units. Note that $F$ is the force component which acts tangential to the filament. The molecular interaction potentials $U_{m}(x)$ are periodic with potential period $\ell$. For kinesin on microtubules, one has $\ell=16$ nm. The lateral currents $J_{m}$ then have the Smoluchowski- or Fokker-Planck form $[22,23]$

$$
J_{m}(x, t) \equiv-D_{o}\left[\frac{\partial}{\partial x} V_{m}(x)+\frac{\partial}{\partial x}\right] P_{m}(x, t)
$$

where the parameter $D_{o}$ represents the small-scale diffusion coefficient.

The transition current densities $I_{m}$ depend on the transition rates $\Omega_{m n}=$ $\Omega_{m n}(x)$ from state $m$ to state $n$ and have the generic form

$$
I_{m}(x, t) \equiv \sum_{n}^{\prime}\left[-P_{m}(x, t) \Omega_{m n}(x)+P_{n}(x, t) \Omega_{n m}(x)\right]
$$

where the prime at the summation sign indicates that $n$ is restricted to $n \neq m$. The transition rates $\Omega_{m n}(x)$ are taken to be localized in space at the discrete set of $K$ positions $x=x_{k}$ with $k=1, \ldots, K$ and $0 \leq x_{1}<\ldots<x_{K}<\ell$ and are expressed as

$$
\Omega_{m n}(x) \equiv \sum_{k} \omega_{m n}\left(x_{k}\right) \ell_{\Omega} \delta\left(x-x_{k}\right)
$$

where $\omega_{m n}\left(x_{k}\right) \geq 0$ are transition rate constants, $\ell_{\Omega} \ll \ell$ represents a molecular 'localization' length, and $\delta(z)$ is Dirac's delta function. The parametrization in terms of delta functions is useful since the nonuniform ratchets or reactiondiffusion models can then be solved analytically. [21]

The ratchet models just defined contain both the applied force $F$ which enters explicitly in (2) and the fuel concentration $\Gamma$ which enters via the transition rate constants $\omega_{m n}\left(x_{k}\right)$ in (5). Explicit solution of these models shows that the dependence on the rate constants and, thus, on the fuel concentration $\Gamma$ exhibits some generic or universal features. These universal features will now be discussed for the motor velocity $v=v(\Gamma)$.

\section{Dependence on fuel concentration}

In a stationary state, the velocity $v$ is related to the total lateral current $J_{\text {tot }} \equiv$ $\sum_{m} J_{m}$ via $v=\ell J_{\text {tot }}$ where $\ell$ is the spatial period of the molecular interaction po-

\footnotetext{
$\ddagger$ These models can also be applied to rotary motors for which $x$ represents an appropriate angular coordinate.
} 
tentials. Thus, the concentration dependence of the motor velocity is determined by the dependence of $J_{\text {tot }}$ on the rate constants $\omega_{m n}\left(x_{k}\right)$.

\subsection{Dependence on rate constants}

First, let us consider a nonuniform ratchet with arbitrary $M$ and $K$ and let us focus on a single rate constant $\omega \equiv \omega_{m n}\left(x_{k}\right)$. Using a transfer matrix method, one can show that all $(M, K)$-models lead to the simple functional dependence

$$
J_{\mathrm{tot}}(\omega)=\frac{a_{0}+a_{1} \omega}{b_{0}+b_{1} \omega}
$$

for the total lateral current $J_{\text {tot }}$ where the coefficients $a_{j}$ and $b_{j}$ with $j=0,1$ depend on the other model parameters.

Likewise, the dependence of the total lateral current on two transition rates, say $\omega_{1}$ and $\omega_{2}$, is found to have the generic multilinear form

$$
J_{\text {tot }}\left(\omega_{1}, \omega_{2}\right)=\frac{a_{0}+a_{1} \omega_{1}+a_{2} \omega_{2}+a_{3} \omega_{1} \omega_{2}}{b_{0}+b_{1} \omega_{1}+b_{2} \omega_{2}+b_{3} \omega_{1} \omega_{2}}
$$

with coefficients $a_{j}$ and $b_{j}$ which again depend on the remaining model parameters.

The functional dependence as given by (7) represents the most general form. In practise, some of the coefficients $a_{j}$ and/or $b_{j}$ in (7) may vanish. This can happen, for instance, if some of the transition rate constants $\omega_{m n}\left(x_{k}\right)$ in (5) are identically zero. The simplest example is given by a 2 -state model with $(M, K)=(2,2)$ and the transition rates

$$
\Omega_{12}(x)=\omega_{1} \ell_{\Omega} \delta\left(x-x_{1}\right) \quad \text { and } \quad \Omega_{21}(x)=\omega_{2} \ell_{\Omega} \delta\left(x-x_{2}\right) .
$$

In this case, the coefficients $a_{0}$ and $b_{0}$ in (7) are identically zero and the coefficients $a_{1}$ and $a_{2}$ vanish for $F=0$.

\subsection{Concentration dependence of motor velocity}

As mentioned, the concentration $\Gamma$ of the fuel molecules enters via the rate constants $\omega_{m n}\left(x_{k}\right)$. First, assume that only one of these rate constants, say $\omega$, is $\Gamma$-dependent. If one assumes that the corresponding chemical reaction follows Michaelis-Menten kinetics [24], one has

$$
\omega^{-1}=\left(c_{1} \Gamma\right)^{-1}+c_{2}^{-1} .
$$

where $c_{1}$ and $c_{2}$ are two $\Gamma$-independent rate constants. If this is inserted into the expression (6) for $J_{\text {tot }}$, one obtains the motor velocity

$$
v(\Gamma)=v_{\text {res }}+\left(v_{\text {sat }}-v_{\text {res }}\right) \frac{\Gamma}{\Gamma_{*}+\Gamma}
$$


with the residual velocity $v_{\text {res }} \equiv v(\Gamma=0)$ and the saturation velocity $v_{\text {sat }} \equiv v(\Gamma=$ $\infty)$. The same $v(\Gamma)$-relationship may also apply if the motor cycle involves two $\Gamma$-dependent rate constants $\omega_{1}(\Gamma)$ and $\omega_{2}(\Gamma)$. One example is provided by the 2 -state model with the transition rates $(8)$ when both $\omega_{1}(\Gamma)$ and $\omega_{2}(\Gamma)$ are related to $\Gamma$ via a Michaelis-Menten-type relation as in (9).

On the other hand, if the total lateral current $J_{\text {tot }}\left(\omega_{1}, \omega_{2}\right)$ has the more general form (7), the $\Gamma$-dependence of the motor velocity is given by

$$
v(\Gamma)=v_{\text {res }}+\left(v_{\text {sat }}-v_{\text {res }}\right) \frac{c \Gamma+\Gamma^{2}}{\Gamma_{*}+d \Gamma+\Gamma^{2}}
$$

with two $\Gamma$-independent coefficients $c$ and $d$.

\subsection{Ratchets with $(M, K)=(3,2)$}

Let us now return to the case of two-headed motors. In general, the corresponding ratchet models will be characterized by two locations with enzymatic activity. If each head (i.e., each enzymatic domain) can be activated only at one of these locations, one has only two $\Gamma$-dependent rate constants. One example for such a model is provided by the previously described $(2,2)$ ratchet with the transition rates (8). If both heads can be activated at both locations, one has a ratchet model with four $\Gamma$-dependent rate constants. One such model with $M=3$ internal states and transitions at $K=2$ locations will now be discussed.

As shown in Figs. 2 and 3, the three internal states are now labeled by $m=$ $0,1,2$. The ground state with $m=0$ corresponds to a doubly-bound state of the two-headed motor, i.e., to a state in which both heads are bound to the microtubule. The excited state with $m=1$ corresponds to a state in which one of the heads, say head 1, is unbound while the second head 2 is still bound to the tubule. Likewise, the second excited state with $m=2$ corresponds to a state in which head 2 is unbound and head 1 is bound. Since both heads are taken to be identical (as for dimeric kinesin), the two force potentials $U_{1}(x)$ and $U_{2}(x)$ for the two excited states have the same potential period $\ell$ and are related via $U_{2}(x)=U_{1}(x-\ell / 2)$, see Fig. 2 .

The symmetry between the two heads also implies that the four transition rates from the ground state to the excited states 1 and 2 and back to the ground state must all be periodic with period $\ell$ and must be related via $\Omega_{02}(x)=$ $\Omega_{01}(x-\ell / 2)$ and $\Omega_{20}(x)=\Omega_{10}(x-\ell / 2)$. Thus, one has to specify only two of the four transition rates, say $\Omega_{01}$ and $\Omega_{10}$. The unbinding rate $\Omega_{01}$ depends on two rate constants, $\omega_{1} \equiv \omega_{\text {tr }}$ and $\omega_{2} \equiv \omega_{\text {le }}$, for the trailing and the leading head, respectively, and is given by

$$
\Omega_{01}(x)=\omega_{1} \ell_{\Omega} \delta(x-\ell / 2)+\omega_{2} \ell_{\Omega} \delta(x) .
$$

Likewise, the rebinding rate $\Omega_{10}(x)$ contains two different rebinding rate constants, $\nu_{1} \equiv \nu_{\text {tr }}$ and $\nu_{2} \equiv \nu_{\text {le }}$, and has the form

$$
\Omega_{10}(x)=\nu_{1} \ell_{\Omega} \delta(x-\ell / 2)+\nu_{2} \ell_{\Omega} \delta(x) .
$$




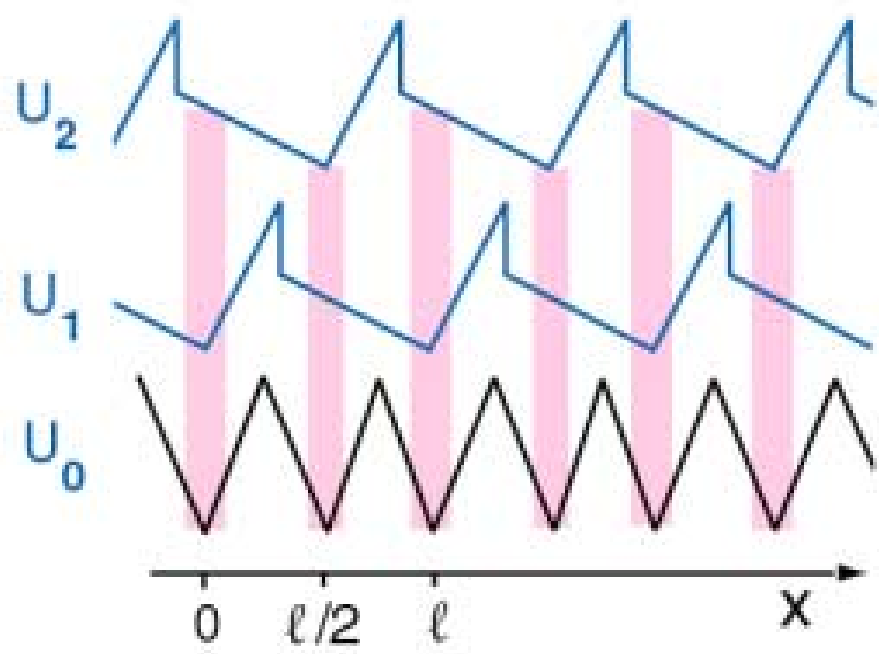

FIG. 2: Ratchet with $(M, K)=(3,2)$ : Functional dependence of the molecular force potentials $U_{m}$ for the three internal states $m=0,1,2$ on the center-of-mass coordinate $x$. The potentials $U_{1}$ and $U_{2}$ for the two excited states have potential period $\ell$ and are related via $U_{2}(x)=U_{1}(x-\ell / 2)$. The shaded vertical stripes represent the localized transitions, compare Fig. 3.

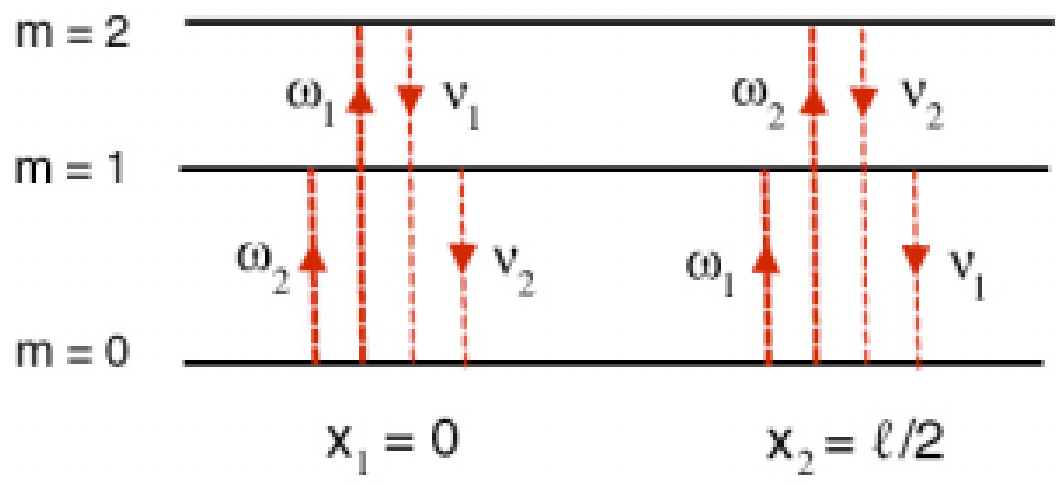

FIG. 3: Ratchet with $(M, K)=(3,2)$ : Transition rates at the two spatial locations $x=x_{1}=0$ and $x=x_{2}=\ell / 2$. The rate constants $\omega_{1}$ and $\omega_{2}$ characterize the unbinding of the trailing and the leading head, respectively, the rate constants $\nu_{1}$ and $\nu_{2}$ the rebinding of these heads to the filament. The unbinding rate constants $\omega_{1}$ and $\omega_{2}$ depend on the fuel concentration $\Gamma$. 
Thus, the 3-state model contains $2 \times 4=8$ transition rates as shown in Fig. 3 . If the $\Gamma$-dependent unbinding rate constants $\omega_{1}$ and $\omega_{2}$ now satisfy $\omega_{1} \sim \omega_{2} \sim \omega(\Gamma)$ with $\omega(\Gamma)$ as given by the Michaelis-Menten relation (9), this $(3,2)$ ratchet leads again to the simple $v(\Gamma)$-relationship as in (10). [21]

\section{Summary and Outlook}

In summary, the functional dependence of the motor velocity $v$ on the concentration $\Gamma$ of the fuel molecules has been analysed for nonuniform ratchet models with $M$ internal states and transitions at $K$ spatial locations. This functional dependence is determined by the number of $\Gamma$-dependent rate constants and can be classified into simple polynomial forms as in (10) and (11). In addition, two subclasses of models for dimeric kinesin with $(M, K)=(2,2)$ and $(M, K)=(3,2)$ lead to the simplest possible relationship (10) which agrees with the experimentally determined fit as given by (1).

It is possible to extend the above analysis by incorporating the limiting case of vanishing fuel concentration for which one must have detailed balance. This leads to a generalized classification scheme for the motor velocity $v=v(\Gamma, F)$, i.e., for the dependence of the velocity on the two control parameters $\Gamma$ and $F$. $[25]$

Acknowledgements I thank Thomas Harms for enjoyable collaborations and Theo M. Nieuwenhuizen for a critical reading of the manuscript.

\section{Glossary: List of symbols}

All symbols are treated as words which are ordered alphabetically.

$D_{o} \quad$ diffusion coefficient in lateral currents $J_{m}$

$F \quad$ applied (tangential) force; a load force corresponds to $F<0$

$\Gamma \quad$ concentration of fuel molecules such as ATP

$\Gamma_{*} \quad$ characteristic intermediate concentration

$I_{m} \quad$ transition current density for internal state $m$

$J_{m} \quad$ lateral current for internal state $m$

$J_{\text {tot }}$ total lateral current

$K \quad$ number of locations for transitions between internal states

$\ell \quad$ period of molecular force potentials

$\ell_{\Omega} \quad$ molecular 'localization' length for transition rates

$M \quad$ number of internal motor states

$\nu \quad$ rebinding rate constant

$P_{m} \quad$ probability density for internal state $m$

$\Omega_{m n}$ spatially dependent transition rate from state $m$ to state $n$

$\omega \quad$ unbinding rate constant

$\omega_{m n}$ transition rate constant from state $m$ to state $n$ 
$x \quad$ spatial coordinate for motor position

$x_{k} \quad$ position at which motor undergoes localized transition

$t \quad$ time

$T \quad$ temperature in energy units

$U_{m} \quad$ molecular force potential for internal state $m$

$V_{m} \quad$ effective force potential as defined in (2).

$v \quad$ motor velocity

$v_{\text {res }} \quad$ residual velocity for small concentration $\Gamma$

$v_{\text {sat }} \quad$ saturation velocity for large concentration $\Gamma$

\section{References}

[1] B. Alberts et al., Essential cell biology: An introduction to the molecular biology of the cell (Garland, New York, 1998).

[2] Maxwell's Demon: Entropy, Information, Computing, edited by H. Leff and A. Rex (Princeton U. P., Princeton, 1990).

[3] J. A. Spudich, Nature 372, 515 (1994).

[4] N. Hirokawa, Science 279, 519 (1998).

[5] J. Howard, A. J. Hudspeth, and R. D. Vale, Nature 342, 154 (1989).

[6] K. Svoboda, C. Schmidt, B. Schnapp, and S. Block, Nature 365, 721 (1993).

[7] K. Svoboda and S. Block, Cell 77, 773 (1994).

[8] W. Hua, E. C. Young, M. L. Fleming, and J. Gelles, Nature 388, 390 (1997).

[9] K. Visscher, M. J. Schnitzer, and S. M. Block, Nature 400, 184 (1999).

[10] Y. Okada and N. Hirokawa, Science 283, 1152 (1999).

[11] A. Mehta et al., Nature 400, 590 (1999).

[12] A. F. Huxley, Biophys. Biophys. Chem. 7, 257 (1957).

[13] S. Leibler and D. A. Huse, J. Cell Biol. 121, 1357 (1993).

[14] R. Astumian and M. Bier, Phys. Rev. Lett. 72, 1766 (1994).

[15] J. Prost, J.-F. Chauwin, L. Peliti, and A. Ajdari, Phys. Rev. Lett. 72, 2652 (1994).

[16] C. Peskin and G. Oster, Biophys. J. 68, 202s (1995).

[17] T. Harms and R. Lipowsky, Phys. Rev. Lett. 79, 2895 (1997). 
[18] L. Schimansky-Geier, M. Kschischo, and T. Fricke, Phys. Rev. Lett. 79, 3335 (1997).

[19] F. Jülicher, A. Ajdari, and J. Prost, Rev. Mod. Phys. 69, 1269 (1997).

[20] M. E. Fisher and A. Kolomeisky, Proc. Natl. Acad. Sci. USA 96, 6597 (1999).

[21] R. Lipowsky and T. Harms, Eur. Biophys. J. 29, 542 (2000).

[22] H. Risken, The Fokker-Planck equation: methods of solution and applications (Springer Verlag, Berlin, 1989).

[23] N. van Kampen, Stochastic processes in physics and chemistry (Elsevier, Amsterdam, 1992).

[24] H. Bisswanger, Enzymkinetik (VCH, Weinheim, 1994).

[25] R. Lipowsky, Phys. Rev. Lett. 85, 4401 (2000). 